\title{
GENERALIZATION OF A RESULT OF BORWEIN AND DITOR
}

\author{
HARRY I. MILLER
}

(Communicated by R. Daniel Mauldin)

\begin{abstract}
D. Borwein and S. Z. Ditor have found a measurable subset $A$ of the real line having positive Lebesgue measure and a decreasing sequence $\left(d_{n}\right)$ of reals converging to zero such that, for each $x, x+d_{n}$ is not in $A$ for infinitely many $n$; thus answering a question of P. Erdös. It will be shown that the result of Borwein and Ditor can be extended using a general 2-place function in place of plus. Related material is also presented.
\end{abstract}

\section{INTRODUCTION}

D. Borwein and S. Z. Ditor [1] have proved the following theorem, answering a question of $P$. Erdös.

Theorem B and D. (1) If $A$ is a measurable set of real numbers with $m(A)>0$ and $\left(d_{n}\right)$ is a sequence converging to zero, then, for almost all $x \in A, x+d_{n} \in A$ for infinitely many $n$.

(2) There is a measurable set $A$, with $m(A)>0$ and a monotonic sequence $\left(d_{n}\right)$ of positive reals converging to zero such that, for each $x, x+d_{n} \notin A$ for infinitely many $n$.

The Baire set analogue of (2) is not true. In [2] it is shown that if $A$ is a subset of the real line satisfying the Baire property (see [3]) and $\left(d_{n}\right)$ is a sequence converging to zero, then the set

$$
A \backslash\left\{x \in A: x+d_{n} \in A \quad \text { for all but finitely many } n\right\}
$$

is of the first Baire category.

Suppose that $A$ is a measurable set of positive reals and $\left(e_{n}\right)$ is a sequence converging to one. It is natural to consider a multiplicative version of the Theorem of Borwein and Ditor. In this paper, we extend the Theorem of Borwein and Ditor to a general result that includes the operations of addition

Received by the editors April 25, 1988.

1980 Mathematics Subject Classification (1985 Revision). Primary 28A05, 26A21.

The research for this paper was supported by the Scientific Research Fund of Bosna and Herzegovina. 
and multiplication as special cases by using general 2-place functions that satisfy appropriate conditions.

\section{RESUlts}

Our first theorem, an extension of part (2) of the Theorem of Borwein and Ditor, is the main result of this paper.

Theorem 1. If $f: R \times R \rightarrow R$ (the real line) is a function satisfying the following conditions:

(a) there exists an $e \in R$ such that $f(x, e)=x$ for every $x \in R$;

(b) $f_{x}$ and $f_{y}$ (partial derivatives) exist and are continuous in a neighborhood of $\left(x_{0}, e\right)$, for some $x_{0} \in R$;

(c) $f_{y}\left(x_{0}, e\right)>0$,

then there exists a strictly decreasing sequence $\left(e_{n}\right)$ of real numbers converging to $e$ and a measurable subset $A$ of $R$ with $m(A)>0$ such that, for all $x \in R$, $f\left(x, e_{n}\right) \notin A$ for infinitely many $n$.

Proof. By (a) and (c), $f_{y}\left(x_{0}, e\right)=a>0$ and $f_{x}\left(x_{0}, e\right)=1$. Therefore, by (b), there exist $x_{1}>x_{0}$ and $y_{1}>e$ such that $f_{x}$ and $f_{y}$ exist and are continuous on the closed rectangular body $T$ having corners at $\left(x_{0}, e\right),\left(x_{0}, y_{1}\right),\left(x_{1}, y_{1}\right)$, $\left(x_{1}, e\right), f_{y}>a / 2$ and $f_{x}>0$ on $T$. Let $t=y_{1}-e$. There exists an integer $n_{0}, n_{0}>2$ such that $(a / 2)(t / 2)>\left(x_{1}-x_{0}\right) / n_{0}$. Divide $\left[x_{0}, x_{1}\right]$ into $n_{0}$ abutting closed intervals, each of length $\left(x_{1}-x_{0}\right) / n_{0}$; denote them by $I_{1}, I_{2}, \ldots, I_{n_{0}}$. Let $J_{1}, J_{2}, \ldots, J_{n_{0}}$ be $n_{0}$ open intervals of equal length satisfying:

(i) $J_{i} \subset I_{i}$ for $i=1,2, \ldots, n_{0}$.

(ii) $J_{i}$ and $I_{i}$ have the same right-hand end points for each $i=1,2, \ldots$, $n_{0}$.

(iii) The set $A_{1}=\left[x_{0}, x_{1}\right] \backslash\left(\bigcup_{i=1}^{n_{0}} J_{i} \cup\left\{x_{1}\right\}\right)$ has measure greater than (0.9) $\left(x_{1}-x_{0}\right)$.

Then, clearly, $A_{1}$ can be written as $A_{1}=\bigcup_{i=1}^{n_{0}} H_{i}$; where the $H_{i}$ 's are closed, disjoint intervals of equal length. Since $f_{x}$ is bounded on $T$, by the mean value theorem for derivatives applied to $f_{x}$, there exists a $d_{1}>0$ such that if $K$ is a closed subinterval of $\left[x_{0}, x_{1}\right]$ of length less than $d_{1}$ and $e<\bar{e}<y_{1}$, then the closed interval $f(K, \bar{e})=\{f(x, \bar{e}): x \in K\}$ has length less than $u_{1} / 2$, where $u_{1}$ is the common length of the $J_{i}$ 's.

Let $m_{1}, m_{1}>2$, be an integer satisfying the inequality $\min \left(d_{1},(a / 2)\left(t / 2^{2}\right)\right)$ $>\left(x_{1}-x_{0}\right) / m_{1} n_{0}$. Divide each of the closed intervals $H_{i}$ into $m_{1}$ abutting closed intervals of equal length; denote these intervals by $H_{i 1}, H_{i 2}, \ldots, H_{i m_{1}}$.

Suppose $K$ is an interval in the collection $\left\{H_{i j}: 1 \leq i \leq n_{0}, 1 \leq j \leq m_{1}\right\}$, say $K=H_{i j}$. By the mean value theorem for derivatives applied to $f_{y}$ and the definition of $n_{0}$, there exists an $e_{K}, e<e_{K}<e+t / 2$, such that $L_{J_{i}}<$ $f\left(L_{K}, e_{K}\right)<M_{J_{i}}$, where $L_{J_{i}}$ and $L_{K}$ denote the left end points of $J_{i}$ and 
$K$ respectively and $M_{J_{i}}$ denotes the midpoint of $J_{i}$. Furthermore, since the length of $K$ is less than $\left(x_{1}-x_{0}\right) / m_{1} n_{0}$, which in turn is less than $d_{1}$, it follows that $f\left(K, e_{K}\right)$ has length less than $u_{1} / 2$ and hence $f\left(K, e_{K}\right) \subset J_{i}$. Of course, for each $K, e_{K}$ is highly nonunique; namely, if $e_{K^{\prime}}$ is sufficiently close to $e_{K}$ than $f\left(K, e_{K^{\prime}}\right) \subset J_{i}$ still holds.

Therefore, there exist $m_{1} n_{0}$ distinct points $\left\{e_{1 i}\right\}_{i=1}^{m_{1} n_{0}}$, each strictly between $e$ and $e+t / 2$, such that for each $x \in A_{1}$, there exists a point $e_{x} \in\left\{e_{1 i}: i=\right.$ $\left.1,2, \ldots, m_{1} n_{0}\right\}$ such that $f\left(x, e_{x}\right) \notin A_{1}$.

Suppose now that $\left\{J_{i j}: 1 \leq i \leq n_{0}, 1 \leq j \leq m_{1}\right\}$ is a collection of open intervals of equal length satisfying:

(iv) $J_{i j} \subset H_{i j}$ for all $1 \leq i \leq n_{0}$ and $1 \leq j \leq m_{1}$.

(v) $J_{i j}$ and $H_{i j}$ have the same right-hand end points for all $1 \leq i \leq n_{0}$ and $1 \leq j \leq m_{1}$.

(vi) $A_{2}=\bigcup_{i=1}^{n_{0}}\left(H_{i} \backslash\left(\bigcup_{j=1}^{m_{1}} J_{i j} \cup\left\{b_{i}\right\}\right)\right)$ has measure greater than (0.9) $\left(x_{1}-x_{0}\right)$, where $b_{i}$ is the right end point of $H_{i}$ for each $1 \leq i \leq n_{0}$.

Let $u_{2}$ denote the common length of the $J_{i j}$ 's. Again, since $f_{x}$ is bounded on $T$, by the mean value theorem there exists a $d_{2}>0$ such that $f(K, \bar{e})$ has length less than $u_{2} / 2$ for every closed subinterval $K$ of $\left[x_{0}, x_{1}\right]$ of length less than $d_{2}$ and every $\bar{e}, e<\bar{e}<y_{1}$.

Let $m_{2}, m_{2}>2$ be an integer satisfying the inequality

$$
\min \left(d_{2},(a / 2)\left(t / 2^{3}\right)\right)>\left(x_{1}-x_{0}\right) /\left(m_{2} m_{1} n_{0}\right) .
$$

Divide each of the $m_{1} n_{0}$ disjoint closed intervals of equal length making up $A_{2}$ into $m_{2}$ abutting closed intervals of equal length. Denote the $m_{2}$ abutting closed intervals making up $H_{i j}$ by $H_{i j 1}, H_{i j 2}, \ldots, H_{i j m_{2}}$.

Suppose $K$ is an interval in the collection $\left\{H_{i j k}: 1 \leq i \leq n_{0}, 1 \leq j \leq\right.$ $\left.m_{1}, 1 \leq k \leq m_{2}\right\}$, say $K=H_{i j k}$. By the mean value theorem applied to $f_{y}$ and the definition of $m_{1}$, there exists an $e_{K}, e<e_{K}<e+t / 2^{2}$, such that $L_{J_{i j}}<f\left(L_{K}, e_{K}\right)<M_{J_{i j}}$, where $L_{J_{i j}}$ and $L_{K}$ are the left end points of $J_{i j}$ and $K$ respectively and $M_{J_{i j}}$ is the midpoint of $J_{i j}$. Furthermore, since the length of $K$ is less than $\left(x_{1}-x_{0}\right) /\left(m_{2} m_{1} n_{0}\right)$ which in turn is less than $d_{2}$, it follows that $f\left(K, e_{K}\right)$ has length less than $u_{2} / 2$ and hence $f\left(K, e_{K}\right) \subset J_{i j}$.

Therefore, there exist $m_{2} m_{1} n_{0}$ distinct numbers $\left\{e_{2 i}\right\}_{i=1}^{m_{2} m_{1} n_{0}}$, each different from the points in $\left\{e_{1 i}\right\}_{i=1}^{m_{1} n_{0}}$ and such that each number $e_{2 i}$ lies strictly between $e$ and $e+t / 2^{2}$ and such that for each $x \in A_{2}$, there exists a point $e_{x} \in$ $\left\{e_{2 i}\right\}_{i=1}^{m_{2} m_{1} n_{0}}$ such that $f\left(x, e_{x}\right) \notin A_{2}$.

This process can be continued by mathematical induction. In this way we obtain sequences

$$
\left\{m_{i}\right\}_{i=1}^{\infty}, \quad\left\{e_{i j}\right\}_{j=1}^{m_{i} m_{i-1} \cdots m_{2} m_{1} n_{0}}, \quad\left\{A_{i}\right\}_{i=1}^{\infty}
$$

satisfying the following conditions. 
(vii) Each $m_{i}$ is an integer greater than 2.

(viii) All of the numbers $e_{i j}$ are distinct and for each $i \in N$ (the natural numbers), $e_{i j}$ lies strictly between $e$ and $e+t / 2^{i}$ for each $j=$ $1,2, \ldots, m_{i} m_{i-1} \cdots m_{2} m_{1} n_{0}$.

(ix) Each set $A_{i}$ is a closed subset of $\left[x_{0}, x_{1}\right], A_{i+1} \subset A_{i}$ for each $i \in N$ and $m\left(A_{i}\right)>(0.9)\left(x_{1}-x_{0}\right)$ for each $i \in N$. For each $x \in A_{i}$, there exists a point $e_{x} \in\left\{e_{i j}\right\}_{j=1}^{m_{i} m_{i-1} \cdots m_{2} m_{1} n_{0}}$, such that $f\left(x, e_{x}\right) \notin A_{i}$.

Let $\left\{e_{n}\right\}$ denote the set $\left\{e_{i j}: i \in N, 1 \leq j \leq m_{i} m_{i-1} \cdots m_{2} m_{1} n_{0}\right\}$ written as a strictly decreasing sequence and let $A=\bigcap_{n=1}^{\infty} A_{n}$.

Clearly $m(A) \geq(0.9)\left(x_{1}-x_{0}\right)>0$ and, by (viii) $\lim _{n \rightarrow \infty} e_{n}=e$. If $x \in A$, then, by (ix), $f\left(x, e_{n}\right) \notin A$ for infinitely many $n$. Finally, if $x \notin A$; since $A$ is closed, $f(x, e)=x$ and $f$ is continuous; it follows that there exists an $n_{x} \in N$ such that $f\left(x, e_{n}\right) \notin A$ for every $n, n \geq n_{x}$.

Remark 1 . Theorem 1 can be extended by replacing condition (a) by the hypothesis that $f_{x}\left(x_{0}, e\right)>0$. Furthermore an $n$-dimensional version of Theorem 1 is valid under appropriate conditions on $f: R^{n} \times R^{n} \rightarrow R^{n}$.

Our next result is an extension of part (1) of Theorem B and D.

Theorem 2. If $A$ is a measurable set of real numbers with $m(A)>0$ and $f: R \times R \rightarrow R$ is a function satisfying the following conditions:

(d) there exists an $e \in R$ such that $f(x, e)=x$ for every $x \in R$;

(e) $f_{x}$ and $f_{y}$ exist and are continuous everywhere;

(f) $f_{y}(x, e)>0$ for all $x \in R$;

and $\left(e_{n}\right)$ is a sequence converging to $e$, then, for almost all $x \in A, f\left(x, e_{n}\right) \in A$ for infinitely many $n$.

Proof. Let $m \in N$ and let $A_{m}$ denote the set $A \cap(-m, m)$. For each $\bar{\varepsilon}>0$, since $A_{m}$ is bounded and measurable, there exists a positive integer $n=n(\bar{\varepsilon})$ and there are disjoint intervals $I_{1}, I_{2}, \ldots, I_{n}$ such that

$$
A_{m}=\left(\bigcup_{k=1}^{n} I_{k} \cup E_{2}\right) \backslash E_{1}
$$

where $E_{1}$ and $E_{2}$ each have outer Lebesgue measure less than $\bar{\varepsilon}$.

Let $\varepsilon>0$. By $(*)$ and the conditions on $f$ there exists a subsequence $\left(n_{k}\right)_{k=1}^{\infty}$ of the natural numbers such that

$$
m\left(A_{m} \backslash f\left(A_{m}, e_{n_{k}}\right)\right)<\varepsilon / 2^{k} \text { for each } k .
$$

From this it follows that $\left\{x \in A_{m}: x\right.$ is not in infinitely many of the sets $\left.f\left(A_{m}, e_{n}\right)\right\}$ has measure less than $\varepsilon$ for each $\varepsilon>0$. Since this holds for each $m \in N$ it follows that for almost all $x \in A, f\left(x, e_{n}\right) \in A$ for infinitely many $n$.

Remark 2. In connection with Theorem 2, see [2].

The Baire set analogue of Theorem 2 is not true. In fact the following holds. 
Theorem 3. If $A$ is a Baire subset of $R$ and $f: R \times R \rightarrow R$ is a function satisfying (d), (e) and (f) and $\left(\mathrm{e}_{n}\right)$ is a sequence converging to $e$, then the set $A \backslash\left\{x \in A: f\left(x, e_{n}\right) \in A\right.$ for all but finitely many $\left.n\right\}$ is of the first Baire category. Proof. This result is immediate by the properties of $f$ and the fact that $A$ has the form $A=(G \backslash P) \cup Q$, where $G$ is open and $P$ and $Q$ are sets of the first category.

\section{REFERENCES}

1. D. Borwein and S. Z. Ditor, Translates of sequences in sets of positive measure, Canad. Math. Bull. 21 (1978), 497-498.

2. H. I. Miller, On certain transformations of sets, Akad. Nauka Umjet. Bosne Hercegov. Rad. LXXVIII, 5-10.

3. J. C. Oxtoby, Measure and Category, Springer-Verlag, Berlin and New York, 1970.

Department of Mathematics, University of Sarajevo 71000, Yugoslavia 